\title{
Circulating vaspin levels and nutritional status and insulin resistance in polycystic ovary syndrome
}

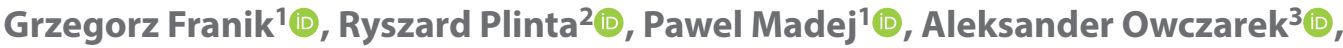 \\ Maria Bozentowicz-Wikarek ${ }^{4}{ }^{\infty}$, Jerzy Chudek ${ }^{4,5}{ }^{\mathbb{D}}$, Violetta Skrzypulec-Plinta ${ }^{6}{ }^{\mathbb{B}}$, \\ Magdalena Olszanecka-Glinianowicz ${ }^{7}$ (D)
}

\author{
${ }^{1}$ Department of Gynecological Endocrinology, Faculty of Medical Sciences in Katowice, Medical University of Silesia, Poland \\ ${ }^{2}$ Chair of Physiotherapy, School of Health Science in Katowice, Medical University of Silesia, Poland \\ ${ }^{3}$ Department of Statistics, Department of Instrumental Analysis, Faculty of Pharmaceutical Sciences in Sosnowiec, \\ Medical University of Silesia in Katowice, Poland \\ ${ }^{4}$ Pathophysiology Unit, Department of Pathophysiology, Faculty of Medical Sciences in Katowice, \\ Medical University of Silesia, Poland \\ ${ }^{5}$ Department of Internal Medicine and Oncological Chemotherapy, School of Medicine in Katowice, \\ Medical University of Silesia, Poland \\ ${ }^{6}$ Women's Health Chair, School of Health Science in Katowice, Medical University of Silesia, Poland \\ ${ }^{7}$ Health Promotion and Obesity Management Unit, Department of Pathophysiology, \\ Faculty of Medical Sciences in Katowice, Medical University of Silesia, Poland
}

\begin{abstract}
Objectives: The study aimed to assess the associations between circulating vaspin levels and nutritional status (assessed on tha basis of $\mathrm{BMI}$ ) as well as insulin resistance in PCOS.

Material and methods: Eighty-seven PCOS women, 48 obese and 39 normal weight, were enrolled in the cross-sectional study. Seventy-two Non-PCOS women, 41 obese and 31 normal weight, constituted a control group. Body mass, height and waist circumference as well as body composition by bioimpedance were measured. In the morning (16h after the last meal) we determined: serum glucose, insulin, androgens, gonadotropin (LH, FSH) and sex hormone-binding globulin (SHBG) as well as plasma vaspin levels. Standard HOMA-IR formula was used to assess insulin resistance (IR).

Results: Plasma vaspin levels were significantly lower in PCOS, both normal weight and obese, than in Non-PCOS groups. Vaspin levels were similar in normal weight and obese PCOS subgroups. There was no association between plasma vaspin levels and anthropometric parameters in PCOS group. While in Non-PCOS group a negative correlation between plasma vaspin levels and body mass $(r=-0.26 ; p<0.05)$ was found. We did not observe correlations between plasma vaspin levels and serum glucose and insulin concentrations as well as HOMA-IR values, however, in multivariable, stepwise backward regression waist circumference and HOMA-IR values explained $18.0 \%$ of plasma vaspin levels variability in the study subjects. Conclusions: PCOS occurrence is associated with decreased vaspin levels. The influence of nutritional status on vaspin level observed in Non-PCOS is abolished in PCOS women, possibly by more severe insulin resistance.
\end{abstract}

Key words: vaspin; insulin resistance; nutritional status; PCOS

Ginekologia Polska 2020; 91, 5: 251-255

\section{INTRODUCTION}

Vaspin is an adipokine, the member of the serine protease inhibitors family (serpin) [1]. Vaspin mRNA expression was found in subcutaneous and visceral adipose tissue, liver, pancreas, stomach and skin [2-5].
The experimental studies have shown that vaspin increased insulin sensitivity and glucose tolerance as well as decreased food intake $[4,6]$. Expression of vaspin mRNA in rats visceral adipose tissue increased with an excess of body mass and insulin resistance [1]. In addition, factors 
stimulating vaspin expression were leptin and metformin and inhibiting night break in food intake [7].

In the human expression of vaspin mRNA in visceral adipose tissue was proportional to BMI value and body fat percentage. In turn, this expression in subcutaneous adipose tissue was proportional to WHR values and fasting serum insulin levels [2]. The association between plasma vaspin levels and BMI values and insulin resistance was also shown in another study [8]. Plasma vaspin levels were higher in women than in men [8]. It is suggested that this sex difference develops during puberty [3]. However, in subjects with metabolic and cardiovascular disturbances the association between circulating vaspin levels and BMI values, sex and age were not observed $[9,10]$.

It has been suggested that increase vaspin mRNA expression and its secretion are a compensatory mechanism that delays insulin resistance development $[2,8,11]$. However, the results of other studies did not confirm this hypothesis [10-13].

Several projects assessing vaspin levels in PCOS showed inconclusive results. Higher vaspin levels were observed in normal-weight PCOS than in Non-PCOS women [14-16]. On the other hand, there were no differences between overweight/obese PCOS and Non-PCOS women [16]. In addition, serum testosterone levels, FAI values and mean ovary volume or the number of follicles were proportional to vaspin levels. While serum FSH and SHBG levels, as well as insulin sensitivity, were inversely related to vaspin levels [16]. The highest plasma vaspin levels were shown in the phenotype $A$, thus this parameter is considered as the marker of the severity of PCOS [17]. The lack of differences between plasma vaspin levels in adolescents with or without PCOS has also been shown [18]. Similarly, vaspin levels did not differ between normal-weight PCOS and Non-PCOS women in Iranian population, despite higher insulin levels in PCOS group [19]. In turn, treatment with metformin and improvement of the insulin sensitivity caused a decrease in vaspin levels [14]. However, Koiou et al. [16] have shown only a slight effect of treatment with metformin on vaspin levels in normal-weight PCOS women and lack of the impact of moderate weight loss on vaspin levels in overweight and obese PCOS women.

The study aimed to assess the associations between circulating vaspin levels and nutritional status (assessed on the basis of BMI) as well as insulin resistance in PCOS.

\section{MATERIAL AND METHODS}

Eighty-seven PCOS (diagnosed on the basis of Rotterdam ESHRE/ASRM criteria [20]) women, 48 obese and 39 normal-weight, were enrolled in the cross-sectional study. Seventy-two Non-PCOS women, 41 obese and 31 normal weight, constituted the control group. The exclusion criteria included any pharmacotherapy, alcohol and nicotine addiction and changes of body mass over $2 \mathrm{~kg}$ during the last 3-months. The written informed consent was obtained from all subjects. The Bioethical Committee of the Medical University of Silesia approved the study protocol.

Nutritional status was diagnosed on the basis of BMI values in accordance with World Health Organization criteria. Table 1 presents the characteristics of the study and control groups.

The venous blood samples $(15 \mathrm{~mL})$ for laboratory tests were collected in the morning 16 hours after the last meal

Table 1. Patients characteristics'

\begin{tabular}{|c|c|c|c|c|c|c|}
\hline & \multicolumn{3}{|l|}{ PCOS } & \multicolumn{3}{|l|}{ non-PCOS } \\
\hline & $\begin{array}{l}\text { All } \\
(n=87)\end{array}$ & $\begin{array}{l}\text { Normal weight } \\
(\mathrm{n}=39)\end{array}$ & $\begin{array}{l}\text { Obese } \\
(n=48)\end{array}$ & $\begin{array}{l}\text { All } \\
(n=72)\end{array}$ & $\begin{array}{l}\text { Normal weight } \\
(\mathrm{n}=31)\end{array}$ & $\begin{array}{l}\text { Obese } \\
(n=41)\end{array}$ \\
\hline Age [years] & $25.4 \pm 5.5$ & $23.7 \pm 4.5^{++}$ & $26.8 \pm 5.8$ & $26.4 \pm 5.5$ & $23.8 \pm 4.3^{\$ \$ \$}$ & $28.4 \pm 5.6$ \\
\hline Body mass [kg] & $79.4 \pm 26.4$ & $56.9 \pm 11.7^{* * *+++}$ & $97.7 \pm 20.2^{8 \& \&}$ & $78.7 \pm 20.4$ & $59.8 \pm 7.1^{\$ \$ \$}$ & $93.1 \pm 14.6$ \\
\hline BMI $\left[\mathrm{kg} / \mathrm{m}^{2}\right]$ & $28.6(20.8-35.7)$ & $20.6^{* *++++}(19.6-22.7)$ & $35.1^{\& \& \&}(31.3-40.2)$ & $28.5(22.9-33.5)$ & $22.4^{\$ \$ \$}(21.0-24.0)$ & $32.9(30.3-36.7$ \\
\hline Body fat [kg] & $30.2(15.4-42.6)$ & $15.0^{* *++}(12.6-19.7)$ & $40.6^{2 \&}(33.4-56.3)$ & $33.3(19.1-50.4)$ & $18.1^{\$ \$}(14.8-20.6)$ & $49.4(37.5-50.2$ \\
\hline Body fat [\%] & $38.1(27.5-45.7)$ & $26.5^{* * *+++}(24.2-31.0)$ & $44.8^{\& \& \&}(41.9-51.1)$ & $40.6(30.4-48.5)$ & $30.0^{\$ \$ \$}(26.8-33.9)$ & $46.8(42.3-51.4$ \\
\hline Waist circumference $[\mathrm{cm}]$ & $89.8 \pm 18.7$ & $72.6 \pm 7.3^{* * *+++}$ & $103.7 \pm 12.3^{8 \& \&}$ & $87.9 \pm 18.2$ & $70.5 \pm 8.3^{\$ \$ \$}$ & $101.0 \pm 11.3$ \\
\hline Total cholesterol [mg/dL] & $176.3 \pm 34.0$ & $167.7 \pm 28.1^{*}$ & $183.2 \pm 37.0$ & $174.6 \pm 30.6$ & $169.1 \pm 33.3$ & $178.8 \pm 27.5$ \\
\hline LDL- cholesterol[mg/dL] & $105.4 \pm 38.3$ & $93.8 \pm 27.5^{* *}$ & $115.1 \pm 37.6$ & $100.3 \pm 27.1$ & $90.3 \pm 31.9$ & $106.4 \pm 21.9$ \\
\hline HDL- cholesterol [mg/dL] & $45.7 \pm 14.1 \% \%$ & $48.1 \pm 15.1^{\text {*\#\# }}$ & $43.8 \pm 12.9^{\& \& \& \wedge \wedge \wedge}$ & $57.1 \pm 15.2$ & $60.1 \pm 16.3^{\$}$ & $54.8 \pm 14.2$ \\
\hline Triglycerides [mg/dL] & $100.7 \pm 55.2$ & $73.0 \pm 31.7^{* * \# \#}$ & $121.5 \pm 61.4^{8 \& \wedge \wedge}$ & $80.1 \pm 32.1$ & $67.2 \pm 26.7^{\$ \$}$ & $89.9 \pm 32.6$ \\
\hline Glucose $[\mathrm{mmol} / \mathrm{L}]$ & $5.1 \pm 0.8 \% \%$ & $4.9 \pm 0.7^{\# \#}$ & $5.3 \pm 0.9^{\& \& \wedge \wedge}$ & $4.7 \pm 0.4$ & $4.7 \pm 0.5$ & $4.7 \pm 0.4$ \\
\hline Insulin $[\mu \mid \mathrm{U} / \mathrm{mL}]$ & $10.6^{\% \%}(7.8-15.1)$ & $8.4^{* *}(6.0-10.6)$ & $12.9^{\& \& \wedge \wedge}(9.7-18.6)$ & $7.4(5.9-9.5)$ & $6.8(5.6-8.7)$ & $7.8(6.3-10.0)$ \\
\hline HOMA-IR & $2.3^{\%} \%(1.6-3.2)$ & $1.8^{* *}(1.2-2.3)$ & $2.8^{\& \& \wedge \wedge}(1.2-4.1)$ & $1.5(1.2-2.0)$ & $1.5(1.1-1.9)$ & $1.7(1.4-2.2)$ \\
\hline
\end{tabular}

${ }^{*} \mathrm{p}<0.05 ;{ }^{* *} \mathrm{p}<0.01 ;{ }^{* * *} \mathrm{p}<0.001$ normal weight PCOS vs obese PCOS; $\mathrm{\#}<0.05 ;{ }^{\# \#} \mathrm{p}<0.01$; \#\# $\mathrm{p}<0.001$ normal weight $\mathrm{PCOS}$ vs normal weight non-PCOS; ${ }^{+} \mathrm{p}<0.05 ;{ }^{++} \mathrm{p}<0.01$; ${ }^{+++} p<0.001$ normal weight PCOS vs obese non-PCOS; ${ }^{*} p<0.05 ; \& \&<0.01 ; \& \& \& p<0.001$ obese PCOS vs normal weight non-PCOS; ${ }^{\wedge} p<0.05 ;{ }^{\wedge \wedge} p<0.01 ;{ }^{\wedge \wedge} p<0.001$ obese PCOS vs obese non-PCOS; $\$ p<0.05 ; \$ p<0.01 ; \$ \$ p<0.001$ normal weight non- PCOS vs obese non-PCOS; $\% p<0.05 ; \% p<0.01 ; \% \%$ p $<0.001$ all $P C O S$ vs all non-PCOS 
between 3-5 days of the menstrual cycle. Height, body mass and waist circumference were measured. BMI was calculated. The bioimpedance method (Bodystat 1500, Douglas, Isle of Man) was used to the assessment of body composition.

Serum and plasma samples were stored frozen in $-70^{\circ} \mathrm{C}$.

\section{Laboratory procedures}

Calorimetric methods (kits made by Roche, Switzerland) were used to determine serum glucose and lipids. Fasting serum insulin levels were measured by enzyme-linked immunosorbent assay — ELISA (DRG Instruments $\mathrm{GmbH}$, Marburg, Germany) with a lower limit of detection of $1.76 \mu \mathrm{IU} / \mathrm{mL}$. The insulin resistance was assessed on the basis of HOMA-IR = fasting concentration of insulin $(\mu \mathrm{lU} / \mathrm{mL}) \times$ fasting concentration of glucose $(\mathrm{mmol} / \mathrm{L}) / 22.5$.

The ELISA (DRG Instruments GmbH, Marburg, Germany) method was also used to determine concentrations of gonadotropin $(\mathrm{FSH}, \mathrm{LH})$, prolactin ( $\mathrm{PRL})$, estradiol $\left(\mathrm{E}_{2}\right)$, androgens (testosterone, free testosterone, androstenedione, DHEA-S) as well as plasma vaspin levels (BioVendor, Brno, The Czech Republic) with the lower limit of detection of $0.01 \mathrm{ng} / \mathrm{mL}$ and intra-assay coefficient variation $7.6 \%$ and inter-assay coefficients variation $7.65 \%$.

In addition, with the standard formula, the free androgen index (FAl) was calculated.

\section{Statistic analysis}

STATISTICA 9.0 PL (StatSoft Poland) software and R software environment were used for statistical analysis. There was no missing data in the database. The mean values \pm stan- dard deviation and median with upper and lower quartiles were used for the presentation of the results. The D'Agostino-Pearson test was used to assess the distribution of variables. The Levene test was used to assess the homogeneity of variances. Two-way multivariable analysis of variances with Duncan post-hoc test was used for comparison of quantitative variables. The multivariable linear regression with the backward stepwise procedure was used to assess the associations between variables. Cook's distance values were used for identification of outliers. Testing the residuals for heteroskedasticity was performed using the Cook-Weisberg test. Models calculation was performed including evaluation of multicollinearity, which was assessed with the variance inflation factor (VIF below 5). Additionally, how well it fit in the obtained model was also assessed with the $F$ test and determination coefficient $R^{2}$. Values below 0.05 were considered statistically significant.

\section{RESULTS}

Body mass and BMI values were similar in the corresponding PCOS and Non-PCOS subgroups. Significantly higher glucose and insulin levels as well as HOMA-IR and FAI values and lower HDL cholesterol and SHBG levels were found in PCOS in comparison to Non-PCOS group and obese than normal-weight PCOS subgroups (Tab. 1 and 2).

Plasma vaspin levels were significantly lower in PCOS than in Non-PCOS group. The lower plasma vaspin levels were shown in both PCOS and in the corresponding Non-PCOS subgroups. Similar plasma vaspin levels were found in normal weight and obese PCOS subgroups, while

Table 2. Serum concentrations of hormones and plasma vaspin levels in analyzed groups of PCOS and non-PCOS

\begin{tabular}{|c|c|c|c|c|c|c|}
\hline & \multicolumn{3}{|l|}{ PCOS } & \multicolumn{3}{|l|}{ non-PCOS } \\
\hline & $\begin{array}{l}\text { All } \\
(n=83)\end{array}$ & $\begin{array}{l}\text { Normal weight } \\
(\mathrm{n}=39)\end{array}$ & $\begin{array}{l}\text { Obese } \\
(n=48)\end{array}$ & $\begin{array}{l}\text { All } \\
(n=72)\end{array}$ & $\begin{array}{l}\text { Normal weight } \\
(n=31)\end{array}$ & $\begin{array}{l}\text { Obese } \\
(n=41)\end{array}$ \\
\hline $\mathrm{FSH}[\mathrm{mlU} / \mathrm{mL}]$ & $5.7(4.4-7.4)$ & $5.5(4.5-6.6)$ & $5.9(4.4-8.5)$ & $5.4(3.9-6.8)$ & $5.4(3.5-6.5)$ & $5.5(4.3-7.1)$ \\
\hline $\mathrm{LH}[\mathrm{mlU} / \mathrm{mL}]$ & $10.0 \%(6.6-14.4)$ & $8.2(5.2-12.8)$ & $10.9^{\& \&}(8.2-15.6)$ & $8.0(6.0-12.2)$ & $7.7(6.4-9.2)$ & $9.1(4.0-15.4)$ \\
\hline LH/FSH & $1.7^{\%}(1.1-2.5)$ & $1.6^{*}(1.0-2.4)$ & $1.7^{\wedge}(1.2-2.6)$ & $1.5(0.9-2.5)$ & $1.5(1.1-2.5)$ & $1.5(0.6-2.5)$ \\
\hline PRL [ng/mL] & $5.9 \% \%(4.1-8.3)$ & $4.6^{\#+++}(3.5-7.7)$ & $6.8(4.9-8.5)$ & $8.1(5.2-10.7)$ & $7.8(6.2-12.0)$ & $8.4(4.7-10.2)$ \\
\hline Androstendione [ng/mL] & $2.2(1.5-3.3)$ & $2.8^{* * \#++}(2.1-3.6)$ & $1.9(1.3-2.8)$ & $1.9(1.4-2.8)$ & $2.1(1.4-3.1)$ & $1.8(1.4-2.6)$ \\
\hline DHEA-S $[\mu \mathrm{g} / \mathrm{mL}]$ & $2.7(2.1-3.5)$ & $2.7(2.2-3.5)$ & $2.8(2.1-3.4)$ & $2.6(1.8-3.5)$ & $2.7(2.1-3.9)$ & $2.4(1.7-3.3)$ \\
\hline Total testosterone $[\mathrm{ng} / \mathrm{mL}]$ & $0.7(0.5-0.9)$ & $0.7(0.5-0.9)$ & $0.6(0.5-0.8)$ & $0.6(0.4-0.8)$ & $0.6(0.4-0.8)$ & $0.6(0.4-0.8)$ \\
\hline Free testosterone $[\mathrm{pg} / \mathrm{mL}]$ & $1.8^{\%}(1.1-2.8)$ & $2.1^{* \#+}(1.4-3.1)$ & $1.6(1.1-2.6)$ & $1.4(0.8-2.3)$ & $1.4(0.9-2.3)$ & $1.4(0.8-2.3)$ \\
\hline Estradiol [pg/mL] & $41.9(29.6-66.7)$ & $43.6(32.7-72.6)$ & $40.0(27.1-64.1)$ & $56.5(29.7-90.7)$ & $56.8(34.4-82.7)$ & $54.9(24.6-111.4)$ \\
\hline SHBG [nmol/l] & $23.0 \% \%(14.8-37.3)$ & $34.2^{* *++}(17.3-49.9)$ & $19.0^{2 \&}(10.8-26.7)$ & $30.7(20.4-49.0)$ & $38.6^{\$ \$}(28.2-63.6)$ & $22.9(16.5-47.3)$ \\
\hline FAI & $3.1 \% \%(1.7-4.9)$ & $2.3^{* * \#+}(1.2-4.4)$ & $3.3^{\&}(2.0-5.1)$ & $1.7(1.1-3.1)$ & $1.5^{\$}(0.9-2.2)$ & $2.0(1.3-4.4)$ \\
\hline Vaspin [ng/mL] & $0.13(0.03-0.87)$ & $\begin{array}{l}0.13^{\# \#++++} \\
(0.04-0.87)\end{array}$ & $\begin{array}{l}0.13^{\& \& \& \wedge \wedge \wedge} \\
(0.03-0.53)\end{array}$ & $0.18(0.03-2.07)$ & $0.16^{\$}(0.03-1.38)$ & $0.19(0.06-2.07)$ \\
\hline
\end{tabular}

${ }^{*} \mathrm{p}<0.05 ;{ }^{* *} \mathrm{p}<0.01 ;{ }^{* * *} \mathrm{p}<0.001$ normal weight PCOS vs obese PCOS; $\mathrm{\#}<0.05 ;{ }^{\# \#} \mathrm{p}<0.01 ;{ }^{\# \# \#} \mathrm{p}<0.001$ normal weight PCOS vs normal weight non-PCOS; ${ }^{+} \mathrm{p}<0.05 ;{ }^{++} \mathrm{p}<0.01$; ${ }^{+++} p<0.001$ normal weight PCOS vs obese non-PCOS; ${ }^{\&} p<0.05 ;{ }^{\& \&} p<0.01 ;{ }^{\& \&} \mathrm{p} p<0.001$ obese PCOS vs normal weight non-PCOS; ${ }^{\wedge} p<0.05 ;{ }^{\wedge \wedge} p<0.01 ;{ }^{\wedge \wedge} p<0.001$ obese PCOS vs obese non-PCOS; $\$ p<0.05 ; \$ p<0.01 ; \$ \$ p<0.001$ normal weight non- PCOS vs obese non-PCOS; $\% p<0.05 ; \% p<0.01 ; \% \%$ p $<0.001$ all $P C O S$ vs all non-PCOS 
lower plasma vaspin levels were observed in normal-weight than in obese Non-PCOS subgroups (Tab. 2).

\section{Correlation between plasma vaspin levels and anthropometric parameters and insulin resistance}

We did not observe any association between plasma vaspin levels or any anthropometric parameters in all study groups and the PCOS group. While in Non-PCOS group the negative correlation between plasma vaspin levels and body mass $(r=-0.26 ; p<0.05)$.

There was no correlation between plasma vaspin levels and serum glucose and insulin concentrations as well as HOMA-IR values in both all study groups and both PCOS and Non-PCOS groups analyzed separately.

\section{Multivariable regression analyses}

Multivariable, stepwise backward regression analyses revealed that waist circumference but no other anthropometric parameters, as well as HOMA-IR values, explained $18.0 \%$ of plasma vaspin levels variability.

\section{DISCUSSION}

The results presented in this study demonstrate lower vaspin levels in PCOS women in corresponding, according to nutritional status, Non-PCOS subgroups. In addition, vaspin levels were affected by measures of nutritional status, but only in Non-PCOS subgroup. They were inversely proportional to body mass, and lower in normal weight than obese Non-PCOS.

It should be noted, that it is the first study that showed lower vaspin levels in PCOS women independently from nutritional status. In four published studies higher vaspin levels in PCOS women were described [14-17] and in two there were no differences between PCOS and Non-PCOS subjects $[18,19]$. In addition, we found striking differences in vaspin levels in previously published studies. Only in one study performed in 12 subjects the median of vaspin levels was similar to obtained in our study [14]. While in Turkish subjects mean vaspin levels were more than two times higher in PCOS group and in the control group almost five times lower than in our study [15]. Similar, differences were observed in Greek cohorts [16].

Contrary, to previously published studies [17] in multiple regression analysis we observed an inverse association between vaspin levels and waist circumference in PCOS subjects. In addition, contradictory to the other studies, we did not observe an association between vaspin levels and BMI values $[8,17]$. These differences are difficult to explain. On the one hand, it may be a result of small study group sizes $[14,15]$, the differences in nutritional status between study and control groups (mean BMI $5 \mathrm{~kg} / \mathrm{m}^{2}$ lower in control group) $[16,17]$ and race. On the other hand, it may be the result of different ELISA kits used to vaspin levels measurements, produced by different manufacturers. The lack of studies comparing specificity of kits produced for vaspin levels assessment should be raised.

Furthermore, we did not observe the association between serum glucose and insulin concentrations as well as HOMA-IR values and circulating vaspin levels. It is opposite to some published studies that show that HOMA-IR values were proportional to vaspin levels in PCOS women [17-19] but in accordance with the results of other studies $[15,17]$. The association between insulin resistance or glucose metabolism and circulating vaspin levels is not clear. It should be noted that only Youn et al. [8] shown an association between HOMA-IR and vaspin levels in group with normal glucose tolerance. In addition, Tan et al. [14] found that vaspin synthesis is stimulated by glucose in omental adipocytes. Moreover, vaspin mRNA expression but not its circulating levels were proportional to insulin resistance. Therefore, we hypothesized that increased vaspin levels may be a compensatory mechanism in the development of the early stage of insulin resistance, that run out over time. This hypothesis is partially supported by the observed lower vaspin levels in PCOS subjects and its higher levels in obese than normal-weight Non-PCOS women. However, further studies with follow-up are necessary to confirm our hypothesis.

The main limitations of the study are the small sizes of study subgroups and the lack of the assessment of the body fat visceral and subcutaneous using DEXA or CT scanner. Additionally, only selected adipokine vaspin was analyzed thus the assessment of the association between hormonal disturbances of adipose tissue and it's inflammation was not possible.

\section{CONCLUSIONS}

PCOS occurrence is associated with decreased vaspin levels in young women. The influence of nutritional status on vaspin level observed in Non-PCOS is abolished in PCOS women, possibly by the coexisting insulin resistance.

\section{Acknowledgements}

The study was supported by the grant of Medical University of Silesia KNW-1-105/N/8/O.

\section{REFERENCES}

1. Hida K, Wada J, Eguchi J, et al. Visceral adipose tissue-derived serine protease inhibitor: a unique insulin-sensitizing adipocytokine in obesity. Proc Natl Acad Sci U S A. 2005; 102(30): 10610-10615, doi: 10.1073/pnas.0504703102, indexed in Pubmed: 16030142.

2. Klöting N, Berndt J, Kralisch S, et al. Vaspin gene expression in human adipose tissue: association with obesity and type 2 diabetes. Biochem Biophys Res Commun. 2006; 339(1): 430-436, doi: 10.1016/j. bbrc.2005.11.039, indexed in Pubmed: 16298335.

3. Körner A, Neef M, Friebe D, et al. Vaspin is related to gender, puberty and deteriorating insulin sensitivity in children. Int J Obes (Lond). 2011; 35(4): 578-586, doi: 10.1038/ijo.2010.196, indexed in Pubmed: 20856257. 
4. Klöting $\mathrm{N}$, Kovacs $\mathrm{P}$, Kern $\mathrm{M}$, et al. Central vaspin administration acutely reduces food intake and has sustained blood glucose-lowering effects. Diabetologia. 2011; 54(7): 1819-1823, doi: 10.1007/s00125-0112137-1, indexed in Pubmed: 21465327.

5. Meyer-Hoffert U. Reddish, scaly, and itchy: how proteases and their inhibitors contribute to inflammatory skin diseases. Arch Immunol Ther Exp (Warsz). 2009; 57(5): 345-354, doi: 10.1007/s00005-009-0045-6, indexed in Pubmed: 19688185.

6. Wada J.Vaspin: a novel serpin with insulin-sensitizing effects. Expert Opin Investig Drugs. 2008; 17(3): 327-333, doi: 10.1517/13543784.17.3.327, indexed in Pubmed: 18321232

7. González CR, Caminos JE, Vázquez MJ, et al. Regulation of visceral adipose tissue-derived serine protease inhibitor by nutritional status, metformin, gender and pituitary factors in rat white adipose tissue. J Physiol. 2009; 587(Pt 14): 3741-3750, doi: 10.1113/jphysiol.2009.172510, indexed in Pubmed: 19470778.

8. Youn BS, Klöting N, Kratzsch J, et al. Serum vaspin concentrations in human obesity and type 2 diabetes. Diabetes. 2008; 57(2): 372-377, doi: 10.2337/db07-1045, indexed in Pubmed: 17991760.

9. Aust G, Richter O, Rohm S, et al. Vaspin serum concentrations in patients with carotid stenosis. Atherosclerosis. 2009; 204(1): 262-266, doi: 10.1016/j.atherosclerosis.2008.08.028, indexed in Pubmed: 18848328.

10. Handisurya A, RiedI M, Vila G, et al. Serum vaspin concentrations in relation to insulin sensitivity following RYGB-induced weight loss. Obes Surg. 2010;20(2): 198-203, doi: 10.1007/s11695-009-9882-y, indexed in Pubmed: 19506980.

11. Zvonic S, Lefevre M, Kilroy G, et al. Secretome of primary cultures of human adipose-derived stem cells: modulation of serpins by adipogenesis. Mol Cell Proteomics. 2007; 6(1): 18-28, doi: 10.1074/mcp. M600217-MCP200, indexed in Pubmed: 17018519.

12. Suleymanoglu S, Tascilar E, Pirgon O, et al.Vaspin and its correlation with insulin sensitivity indices in obese children. Diabetes Res Clin Pract. 2009;84(3): 325-328, doi: 10.1016/j.diabres.2009.03.008, indexed in Pubmed: 19356820.

13. Gulcelik NE, Karakaya J, Gedik A, et al. Serum vaspin levels in type 2 diabetic women in relation to microvascular complications. Eur J En- docrinol. 2009; 160(1): 65-70, doi: 10.1530/EJE-08-0723, indexed in Pubmed: 18952766

14. Tan BK, Heutling $\mathrm{D}$, Chen J, et al. Metformin decreases the adipokine vaspin in overweight women with polycystic ovary syndrome concomitant with improvement in insulin sensitivity and a decrease in insulin resistance. Diabetes. 2008; 57(6): 1501-1507, doi: 10.2337/db08-0127, indexed in Pubmed: 18375437.

15. Cakal $E$, Ustun $Y$, Engin-Ustun $Y$, et al. Serum vaspin and $C$-reactive protein levels in women with polycystic ovaries and polycystic ovary syndrome. Gynecol Endocrinol. 2011; 27(7): 491-495, doi: 10.3109/09513590.2010.501874, indexed in Pubmed: 20626239.

16. Koiou E, Tziomalos K, Dinas K, et al. The effect of weight loss and treatment with metformin on serum vaspin levels in women with polycystic ovary syndrome. Endocr J. 2011; 58(4): 237-246, doi: 10.1507/endocrj. k10e-330, indexed in Pubmed: 21325745.

17. Koiou E, Dinas K, Tziomalos K, et al. The phenotypes of polycystic ovary syndrome defined by the 1990 diagnostic criteria are associated with higher serum vaspin levels than the phenotypes introduced by the 2003 criteria. Obes Facts. 2011; 4(2): 145-150, doi: 10.1159/000327935, indexed in Pubmed: 21577021.

18. Cekmez F, Cekmez Y, Pirgon O, et al. Evaluation of new adipocytokines and insulin resistance in adolescents with polycystic ovary syndrome. Eur Cytokine Netw. 2011; 22(1): 32-37, doi: 10.1684/ecn.2011.0279, indexed in Pubmed: 21411410.

19. Akbarzadeh S, Ghasemi S, Kalantarhormozi M, et al. Relationship among plasma adipokines, insulin and androgens level as well as biochemical glycemic and lipidemic markers with incidence of PCOS in women with normal BMI. Gynecol Endocrinol. 2012; 28(7): 521-524, doi: 10.3109/09513590.2011.650747, indexed in Pubmed: 22309615.

20. Rotterdam ESHRE/ASRM-Sponsored PCOS Consensus Workshop Group, Rotterdam ESHRE/ASRM-Sponsored PCOS consensus workshop group. Revised 2003 consensus on diagnostic criteria and long-term health risks related to polycystic ovary syndrome (PCOS). Hum Reprod. 2004; 19(1): 41-47, doi: 10.1093/humrep/deh098, indexed in Pubmed: 14688154. 